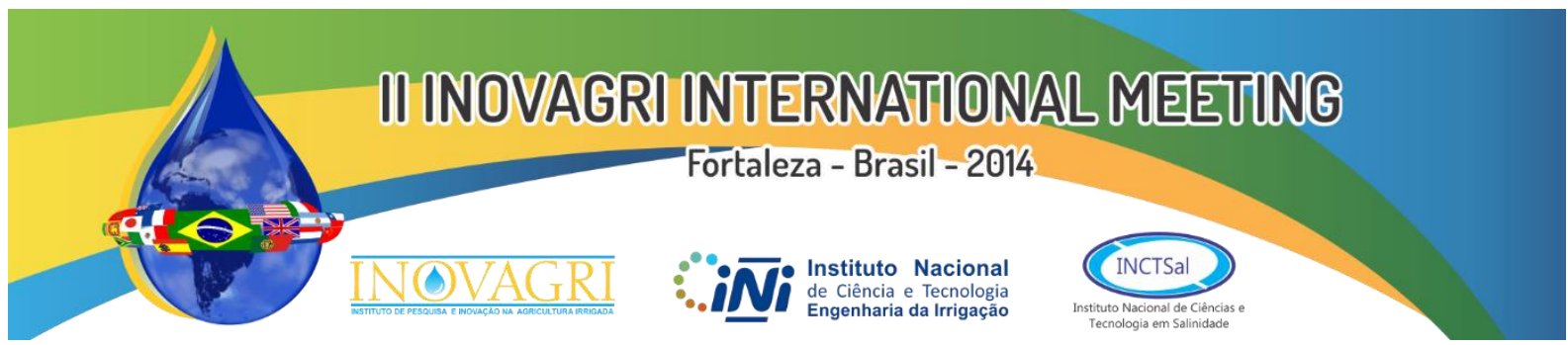

http://dx.doi.org/10.12702/ii.inovagri.2014-a280

\title{
EVAPOTRANSPIRAÇÃO DE REFERÊNCIA PARA DIMENSIONAMENTO DE SISTEMAS DE IRRIGAÇÃO EM PETROLINA, PE
}

\author{
A. O. da Silva ${ }^{1}$, J. S. Correia ${ }^{1}$, L. H. Bassoi ${ }^{2}$, A. H. de C. Teixeira ${ }^{3}$
}

RESUMO: A evapotranspiração é uma variável fundamental para estimar a exigência hídrica das culturas, e também fornece subsídio para o dimensionamento de sistemas de bombeamento, adução e distribuição de água na irrigação. A caracterização da distribuição de frequência da evapotranspiração de referência (ETo), estimada pelo método do tanque classe "A" em Petrolina - PE, foi realizada com base em série histórica de dados de 1963 a 2010, os quais foram agrupados em períodos de 5, 10, 15 e 30 dias. As distribuições gama e normal foram aplicadas aos dados de ETo com probabilidade de 95, 90, 75, 50, 25, 10 e 5\%, e foram aptas para representar os dados de evapotranspiração de referência para os períodos acumulados de 10,15 e 30 dias. Os valores de ETo (11,06 e 11,07 $\mathrm{mm} \mathrm{dia}^{-1}$, distribuição gama e normal, respectivamente) para o mês de outubro no período acumulado de 15 dias com probabilidade de $75 \%$, foram os mais indicados para o dimensionamento da lâmina diária máxima de projetos de sistemas de irrigação.

PALAVRAS-CHAVE: Probabilidade, frequência de distribuição, semiárido.

\section{REDERENCE EVAPOTRANSPIRATION FOR DESIGN OF IRRIGATION SYSTEMS IN PETROLINA, BRAZIL}

\begin{abstract}
Evapotranspiration is a key variable to estimate crop water requirement, to support the design of pumping systems, supply and distribution of water for irrigation. The distribution frequency of reference evapotranspiration (ETo) estimated by class A pan in Petrolina, State of Pernambuco, Brazil, was characterized based on 1963 - 2010 data serie, grouped in periods of 5,10,15 and 30 days. Gamma and normal distributions were applied to the ETo data with a probability of 95, 90,75, 50, 25, 10 and 5\%, and were able to represent the ETo data for the cumulative periods of 10,15 and 30 days. The ETo values (11.06 and $11.07 \mathrm{~mm} \mathrm{day}^{-1}$, normal and gamma distribution, respectively) for the month of October in the period from 15 days with $75 \%$ probability were the most suitable for the scaling of the maximum daily irrigation depth of irrigation system designs.
\end{abstract}

KEYWORDS: Probability, distribution frequency, semi-arid.

\section{INTRODUÇÃO}

A agricultura apresenta grande dependência das condições climáticas atuantes nas regiões de cultivo, sendo influenciada de maneira significativa pelos diversos fatores meteorológicos existentes, dentre estes fatores destacam-se a precipitação, radiação solar, vento, evapotranspiração, etc. Por isso, é fundamental estudar a influência das suas variações sobre as diferentes estratégias de uso do sistema agrícola, de modo a apresentar subsídios para o processo de tomada de decisão e otimizar o planejamento das atividades agrícolas (Frizzone \& Andrade Júnior, 2005).

$\mathrm{Na}$ agricultura irrigada, o conhecimento da evapotranspiração é de grande importância na estimativa das necessidades hídricas das culturas, bem como para o manejo racional dos recursos

\footnotetext{
${ }^{1}$ Doutorando, bolsista CAPES/CNPq, UNESP/FCA, Botucatu - São Paulo. Email: alexsandro_oliveira01@ hotmail.com; linajua@hotmail.com

${ }^{2}$ Pesquisador, Embrapa Semiárido, Petrolina - Pernambuco. Email: luis.bassoi@embrapa.br

${ }^{3}$ Pesquisador, Embrapa Monitoramento por Satélite, Campinas - São Paulo. E.mail: heriberto.teixeira@embrapa.br
}
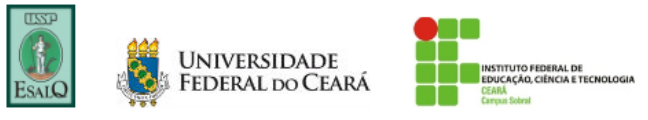
hídricos (Frizzone, 2007; Carvalho \& Oliveira, 2012). Segundo Saad et al., (2002) a estimativa da necessidade hídrica das culturas é de fundamental importância para dimensionar o sistema de adução, de distribuição e de aplicação de água, característicos de cada método de irrigação. Porém, existem dificuldades para uma estimativa adequada da evapotranspiração da cultura, pois a diversidade de métodos de estimativa da evapotranspiração de referência (ETo) e a dificuldade para seu cálculo, devido a grande quantidade de parâmetros meteorológicos exigidos, além de breves históricos destes na maioria das propriedades, dificultam uma estimativa mais precisa. Por isso a utilização de métodos alternativos para estimativa da ETo, como o tanque classe "A", devem ser utilizados para o planejamento da agricultura irrigada, devido principalmente a sua fácil aplicação, além de possuir em alguns casos, dados históricos maiores, devido ao período longo de uso das estações meteorológicas convencionais no Brasil (Trajkovic \& Kolakovic, 2010; Sentelhas \& Folegatti, 2003).

A utilização de simulações com o uso de dados históricos para o planejamento da irrigação é uma técnica adotada com a finalidade de fazer previsões de fenômenos meteorológicos, consistindo em simular uma ocorrência futura de possíveis valores (Fietz et al., 1997). Segundo Frizzone \& Andrade Júnior (2005), a técnica de simulação é de grande importância, pois séries de dados climáticos disponíveis para efetuar estudos envolvendo as inter-relações clima versus sistema agrícola são muito pequenas, levando a obtenção de resultados tendenciosos. Doorenbos \& Pruitt (1997) advertem que o uso de dados climáticos médios em equações combinadas pode conduzir a erros significativos, comprometendo todo planejamento realizado.

Back (2007), Saad et al., (2002) e Silva et al., (1998) sugerem uma análise da probabilidade de ocorrência dos valores históricos de ETo para fins de dimensionamento de irrigação, porém estes autores afirmam que a escolha do nível de probabilidade deve basear-se em uma análise econômica, considerando os prejuízos associados à redução da quantidade e da qualidade da produção, decorrentes da deficiência hídrica, e o aumento de custos do sistema para satisfazer níveis mais elevados de probabilidade. Doorembos \& Pruitt (1997) consideram que na maioria das regiões irrigadas os níveis de probabilidade estejam entre $75 \%$ e $80 \%$ sendo inviável economicamente a utilização de probabilidades superiores a 90\%, mesmo em condições semiáridas. No Semiárido brasileiro, que apesar do baixo regime de precipitação, apresenta épocas chuvosas nos primeiros meses do ano. $\mathrm{O}$ objetivo deste trabalho foi o de analisar a frequência e a distribuição dos dados de evapotranspiração de referência pelo método do tanque classe "A", na região de Petrolina - PE, e seu ajustamento aos modelos probabilísticos normal e gama.

\section{MATERIAIS E MÉTODOS}

Foram utilizados os dados diários de evaporação, velocidade do vento e umidade do ar registrados na estação meteorológica da área experimental da Embrapa Semiárido, localizada no perímetro irrigado de Bebedouro em Petrolina-PE (latitude $09^{\circ} 08^{\prime} 10,4$ " S, longitude $40^{\circ} 18$ ' 30,6" W) no período de 1960 a 2013.

A evapotranspiração de referência (ETo) foi calculada pelo método do tanque classe "A" conforme equação 1 e descritos por Doorenbos e Pruitt (1997), nos 12 meses de cada ano estudado. Posteriormente, foram obtidos os valores para os intervalos de cinco (pentadas), dez (decêndios), quinze (quinzenais) e trinta (mensais) dias para os valores de ETo.

em que:

$$
E T o=E C A \times K p(1)
$$

ETo - evapotranspiração de referência $\left(\mathrm{mm} \mathrm{dia}^{-1}\right)$;

ECA - evaporação do tanque $\left(\mathrm{mm} \mathrm{dia}^{-1}\right)$;

$\mathrm{Kp}$ - coeficiente do tanque classe "A" (adimensional).

Os valores do coeficiente do tanque para as condições de Petrolina foram obtidos com base na tabela proposta por Doorenbos \& Pruitt (1977) utilizando-se a equação de regressão ajustada por Snyder (1992):

$$
K p=0,482+0,024 \ln F-0,000376 U+0,0045 U R(2)
$$


em que:

F - distância da área de bordadura em relação ao centro do tanque (m);

$\mathrm{U}$ - velocidade do vento $\left(\mathrm{km} \mathrm{h}^{-1}\right)$;

UR - umidade relativa do ar (\%).

Para verificar a frequência dos valores de ETo, foram calculados os valores esperados de evapotranspiração de referência com probabilidades de 5, 10, 25, 50, 75, 90 e 95\%, utilizando a distribuição normal e a distribuição gama.

A função densidade de probabilidade para a distribuição gama pode ser verificada pela equação 3 (Devore, 2006):

$$
f(x, \alpha, \beta)=\left\{\begin{array}{lr}
\frac{1}{\beta^{\alpha} \Gamma(\alpha)} X^{\alpha-1} e^{-\frac{x}{\beta}} & x \geq 0 \\
0 & \text { Caso contrario }
\end{array}\right.
$$

As estimativas dos parâmetros $\alpha$ e $\beta$ foram efetuadas pelo método da máxima verossimilhança, por meio das equações 4 a 8 (Assis et al., 1996):

$$
\begin{gathered}
\alpha=\frac{1}{4 A}\left(1+\sqrt{1+\frac{4 A}{3}}\right)(4) \\
\beta=\frac{\bar{X}}{\alpha}
\end{gathered}
$$

em que:

$\alpha$ - parâmetro de forma

$\beta$ - parâmetro de escala da distribuição gama, sendo:

$$
\begin{gathered}
A=\ln \overline{\mathrm{X}}-\mathrm{X}_{\mathrm{g}}(6) \\
\bar{X}=\frac{1}{N} \sum_{i=1}^{N} \mathrm{x}_{\mathrm{i}}(7) \\
X_{g}=\frac{1}{N} \sum_{i=1}^{N} \ln \left(\mathrm{x}_{\mathrm{i}}\right)
\end{gathered}
$$

em que:

$\mathrm{X}$ - média aritmética da amostra;

$\mathrm{X}_{\mathrm{g}}$ - média geométrica da amostra;

$\mathrm{N}$ - é o número de anos observados.

A função densidade de probabilidade da distribuição normal pode ser representada pela equação 9 (Araújo et al., 2010; Martin et al., 2007):

em que:

$$
f(x)=\frac{1}{\sigma \sqrt{2 \pi}} e\left(-\frac{(x-\mu)^{2}}{2 \sigma^{2}}\right) ; \text { para }-\infty<+\infty
$$

$\mu$ - média;

$\sigma$ - desvio padrão da variável aleatória;

Para verificar o ajuste das distribuições de probabilidade aos dados de evapotranspiração de referência, foi realizado o teste de Kolmogorov-Smirnov, a 5\% de significância, conforme equações (Kite,1978; Back, 2007; Saad et al., 2002):

$$
D_{n}^{+}=\max \left(F_{n}(x)-F(x)\right)(10)
$$




$$
\begin{gathered}
D_{n}^{-}=\max \left(F(x)-F_{n}(x)\right)(11) \\
F_{n}(x)=\frac{1}{n} \sum_{i=1}^{n} \begin{cases}1, & \text { se } y_{i}<x \\
0, & \text { Caso contrário }\end{cases}
\end{gathered}
$$

em que:

$\mathrm{F}_{\mathrm{n}}(\mathrm{x})$ - é a função distribuição acumulada para $n$ observações; $\mathrm{F}(\mathrm{x})$ - é a função de distribuição em hipótese.

\section{RESULTADOS DISCUSSÃO}

Na Tabela 1, encontram-se os valores dos desvios máximos entre os valores observados e os valores estimados de evapotranspiração de referência para a distribuição normal e gama. Observa-se que as distribuições estudadas no período de 5 dias não apresentaram ajuste para a maioria dos meses em estudo, rejeitando-se assim a hipótese Ho ao nível de significância de 5\%. Para os demais períodos (10, 15 e 30 dias) as distribuições de frequências estudadas apresentaram um ajuste adequado para o teste de aderência proposto, aceitando-se assim a hipótese Ho para estas condições. Segundo Silva et al., (1998), em estudos sobre a distribuição e frequência da evapotranspiração em Cruz das Almas-BA, devido a semelhança entre os ajustes das distribuições de frequência com relação ao teste de aderência, estes autores recomendam o uso da distribuição normal, devido a sua maior facilidade de aplicação. Porém, alguns autores como Back (2007) e Saad et al., (2002) recomendam o uso da distribuição beta pois esta apresentou melhores ajustes para as regiões de Urusanga-SC e Piracicaba-SP.

Na Figura 1, estão representados os valores de evapotranspiração de referência calculados para um período acumulado de 15 dias com diferentes níveis de probabilidade para os 12 meses do ano. Observa-se que a maior demanda hídrica foi encontrada para o mês de outubro com valores máximos de 11,86 e $11,71 \mathrm{~mm} \mathrm{dia}^{-1}$ para a distribuição normal (A) e gama (B), com probabilidade de $95 \%$. Os menores valores foram observados para a probabilidade de $5 \%$ apresentando 7,47 e 7,46 mm dia ${ }^{-1}$ para a distribuição normal e gama, respectivamente. Possivelmente a ausência de chuva neste período contribuiu de maneira significativa para o aumento da ETo, fazendo com que a irrigação seja de grande importância neste período.

A Figura 2 apresenta a evapotranspiração de referência para os diferentes níveis de probabilidade de ocorrência para a distribuição normal em função da duração dos períodos estudados para o mês de outubro. Observa-se que houve uma diminuição dos valores da ETo com o aumento do período estudado. Segundo Jensen (1974), o período de máxima exigência hídrica das culturas anuais irrigadas não deve se restringir a um único dia, devendo ser compreendido entre duas ou três semanas. Com relação à probabilidade a ser utilizada para o uso em projetos de irrigação, diversos trabalhos como Back (2007), Saad et al., (2002) e Fietz et al., (1997) recomendam que o valor da evapotranspiração de referência para o dimensionamento de sistemas de irrigação, seja obtido com $75 \%$ de frequência no período de maior demanda de água do ano. Para esta probabilidade os períodos acumulados de 10, 15 e 30 dias (significativos pelo teste de aderência) apresentaram uma ETo de 10,7, 11,06 e 10,64 mm dia ${ }^{-1}$, respectivamente, sendo estes valores superiores aos obtidos por Silva et al., (1998) para a região de Cruz das Almas - BA.

Os valores observados para a distribuição gama (Figura 3) foram semelhantes aos observados na distribuição normal $(+0,34 \%$, em média) podendo ser ambas as distribuições utilizadas para a determinação da frequência da ETo. Para os períodos acumulados de 10,15 e 30 dias a distribuição gama apresentou ETo de 10,7, 11,07 e 10,69 $\mathrm{mm} \mathrm{dia}^{-1}$, respectivamente, para a probabilidade de $75 \%$.

\section{CONCLUSÃO}

As distribuições normal e gama foram aptas para representar os dados de evapotranspiração de referência para os períodos acumulados de 10,15 e 30 dias. A evapotranspiração de referência apresentou tendência de redução de acordo com o aumento do período acumulado, sendo recomendado o período acumulado de 15 dias com probabilidade de $75 \%$ para o dimensionamento de sistemas de irrigação para a região de Petrolina-PE. 
A. O. da Silva et al.

\section{REFERÊNCIAS BIBLIOGRÁFICAS}

ARAÚJO, E. M.; SILVA, I. N.; OLIVEIRA, J. B.; CAVALCANTE JÚNIOR, E. G.; ALMEIDA, B. M. Aplicação de seis distribuições de probabilidade a séries de temperatura máxima em Iguatu-CE. Revista Ciência Agronômica, v.41, n.1, p. 36-45, 2010.

ASSIS, F.N.; ARRUDA, H.V.; PREREIRA, A.R. Aplicação de estatística à climatologia. Pelotas, Editora Universitária, Universidade Federal de Pelotas, 1996 161p.

BACK, A. J. Variação da evapotranspiração de referência calculada em diferentes intervalos de tempo. Engenharia Agrícola, v. 27, n.1, p.139-145, 2007. http://dx.doi.org/10.1590/S0100$\underline{69162007000100007}$

CARVALHO, D. F.; OLIVEIRA, L. F. C. Planejamento e manjo da água na agricultura irrigada. Viçosa: Ed. UFV, 2012, 240p.

DEVORE, J. L. Probabilidade e estatística para engenharia e ciências. São Paulo: Thomson, 2006. 692p.

DOORENBOS, J.; PRUITT, W.O. Crop water requirements. Roma: FAO, 1997. 212p.

FRIZZONE, J. A. Planejamento da irrigação com uso de técnicas de otimização. Revista Brasileira de Agricultura Irrigada, v.1, n.1, p.24-49, 2007. http://dx.doi.org/10.7127/rbai.v1n100107

FRIZZONE, J. A.; ANDRADE JÚNIOR, A. S. Planejamento de irrigação: Análise de decisão e investimentos. Brasília: Embrapa Informação Tecnológica, 2005. 626p.

FIETZ, C. R.; FRIZZONE, J. A.; FOLEGATTI, M. V.; PINTO, J. M. Probabilidade de ocorrência da evapotranspiração de referência na região de Dourados, MS. Ciência Rural, v. 27, n.2, p. 207-210, 1997. http://dx.doi.org/10.1590/S0103-84781997000200006

JENSEN, M.E. Consumptive use of water and irrigation water requirements. New York: ASCE, 1974. $215 \mathrm{p}$.

KITE, G.H. Frequency and risk analyses in hydrology. Fort Collins: Water Resources Publications, 1978. $224 \mathrm{p}$.

MARTIN, T. N.; STORCK, L.; DOURADO NETO, D. Simulação estocástica da radiação fotossinteticamente ativa e da temperatura do ar por diferentes métodos. Pesquisa Agropecuária Brasileira, v. 42, n. 09, p. 1211-1219, 2007. http://dx.doi.org/10.1590/S0100-204X2007000900001

SAAD, J. C.; BISCARO, G. A.; DELMANTO JÚNIOR, O.; FRIZZONE, J. A. Estudo da distribuição da evapotranspiração de referência visando o dimensionamento de sistemas de irrigação. Irriga, v. 7, n. 1, 2002.

SENTELHAS, P. C.; FOLEGATTI, M. V. Class A pan coefficients (p) to estimate daily reference evapotranspiration. Revista Brasileira de Engenharia Agrícola e Ambiental, v. 7, n.1, p.111-115, 2003. http://dx.doi.org/10.1590/S1415-43662003000100018

SILVA, F. C.; FIETZ, C. R.; FOLEGATTI, M. V.; PEREIRA, F. A. C. Distribuição e frequência da evapotranspiração de referência de Cruz das Almas, BA. Revista Brasileira de Engenharia Agrícola e Ambiental, v.2, n.3, p. 284-286, 1998.

TRAJKOVIC, S.; KOLAKOVIC, S. Comparison of simplified pan-based equations for estimating reference evapotranspiration. Journal of Irrigation and Drainage Engineering, v. 136, 137-140, 2010. http://dx.doi.org/10.1061/(ASCE)IR.1943-4774.0000133 
Tabela 1. Desvios máximos entre as frequências observadas e as frequências estimadas pela distribuição normal e distribuição gama para valores de evapotranspiração de referência em Petrolina-PE.

\begin{tabular}{lllllllll}
\hline \multirow{2}{*}{ Mês } & \multicolumn{7}{c}{ Intervalos (dias) } \\
\cline { 2 - 9 } & \multicolumn{2}{c}{5} & \multicolumn{2}{c}{10} & \multicolumn{2}{c}{15} & \multicolumn{1}{c}{30} \\
\cline { 2 - 8 } & Normal & Gama & Normal & Gama & Normal & Gama & Normal & Gama \\
\hline Jan & $0,074^{*}$ & 0,122 & $0,041^{*}$ & $0,054^{*}$ & $0,080^{*}$ & $0,075^{*}$ & $0,098^{*}$ & $0,085^{*}$ \\
Fev & 0,101 & $0,086^{*}$ & 0,103 & $0,070^{*}$ & $0,066^{*}$ & $0,082^{*}$ & $0,142^{*}$ & $0,070^{*}$ \\
Mar & 0,098 & $0,075^{*}$ & $0,082^{*}$ & $0,092^{*}$ & $0,056^{*}$ & $0,098^{*}$ & 0,679 & 0,689 \\
Abr & $0,089^{*}$ & $0,064^{*}$ & $0,090^{*}$ & $0,060^{*}$ & $0,084^{*}$ & $0,093^{*}$ & $0,085^{*}$ & $0,070^{*}$ \\
Mai & 0,102 & $0,066^{*}$ & $0,035^{*}$ & $0,070^{*}$ & $0,068^{*}$ & $0,043^{*}$ & $0,101^{*}$ & $0,092^{*}$ \\
Jun & $0,079^{*}$ & $0,055^{*}$ & $0,048^{*}$ & $0,071^{*}$ & $0,062^{*}$ & $0,062^{*}$ & $0,081^{*}$ & $0,070^{*}$ \\
Jul & 0,127 & 0,093 & $0,042^{*}$ & $0,055^{*}$ & $0,074^{*}$ & $0,079^{*}$ & $0,055^{*}$ & $0,064^{*}$ \\
Ago & 0,151 & 0,133 & $0,065^{*}$ & $0,086^{*}$ & $0,092^{*}$ & $0,119^{*}$ & 0,247 & 0,350 \\
Set & 0,207 & 0,178 & $0,090^{*}$ & $0,105^{*}$ & $0,112^{*}$ & $0,131^{*}$ & $0,192^{*}$ & 0,232 \\
Out & 0,126 & 0,101 & $0,113^{*}$ & 0,138 & $0,050^{*}$ & $0,067^{*}$ & $0,129^{*}$ & $0,152^{*}$ \\
Nov & $0,064^{*}$ & $0,066^{*}$ & $0,106^{*}$ & 0,122 & $0,085^{*}$ & $0,110^{*}$ & $0,116^{*}$ & $0,137^{*}$ \\
Dez & $0,080^{*}$ & 0,114 & $0,064^{*}$ & $0,094^{*}$ & $0,058^{*}$ & $0,082^{*}$ & $0,078^{*}$ & $0,100^{*}$ \\
\hline
\end{tabular}

* significativo a $5 \%$
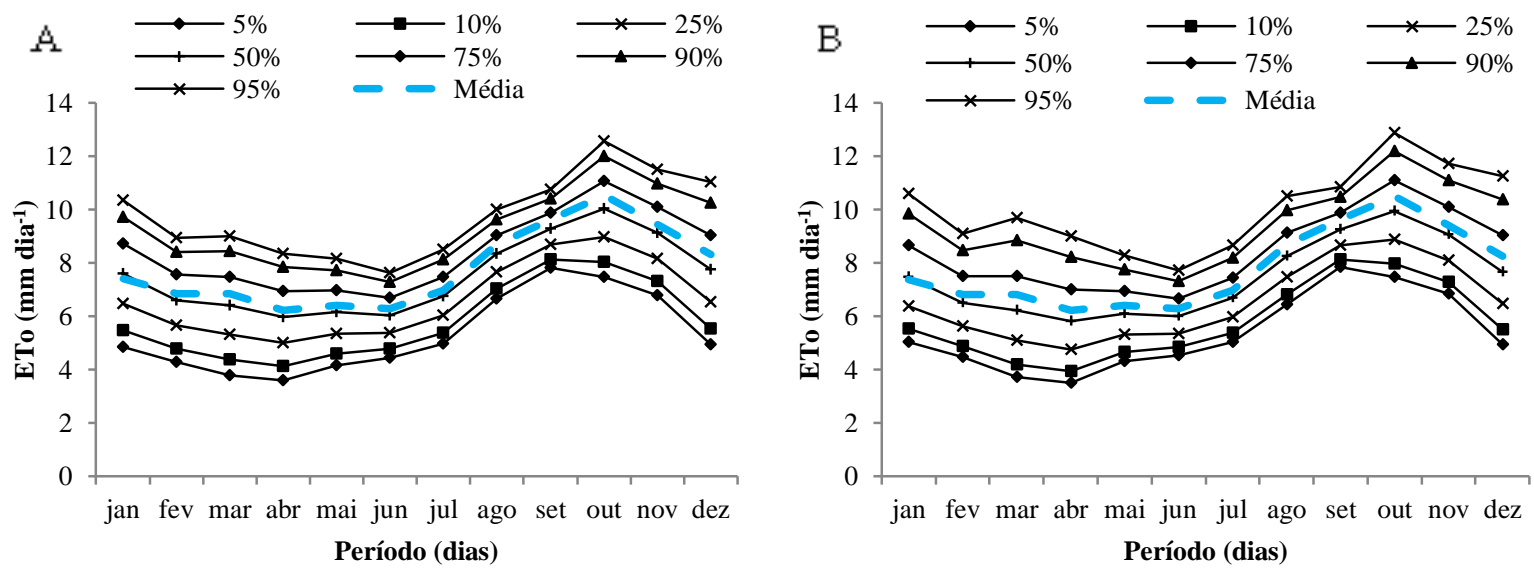

Figura 1. Evapotranspiração de referência calculada em intervalos de 15 dias com probabilidade de 5\%, 10\%, 25\%, 50\%, 75\%, 90\% e 95\% utilizando a distribuição normal (A) e gama (B), em Petrolina-PE.

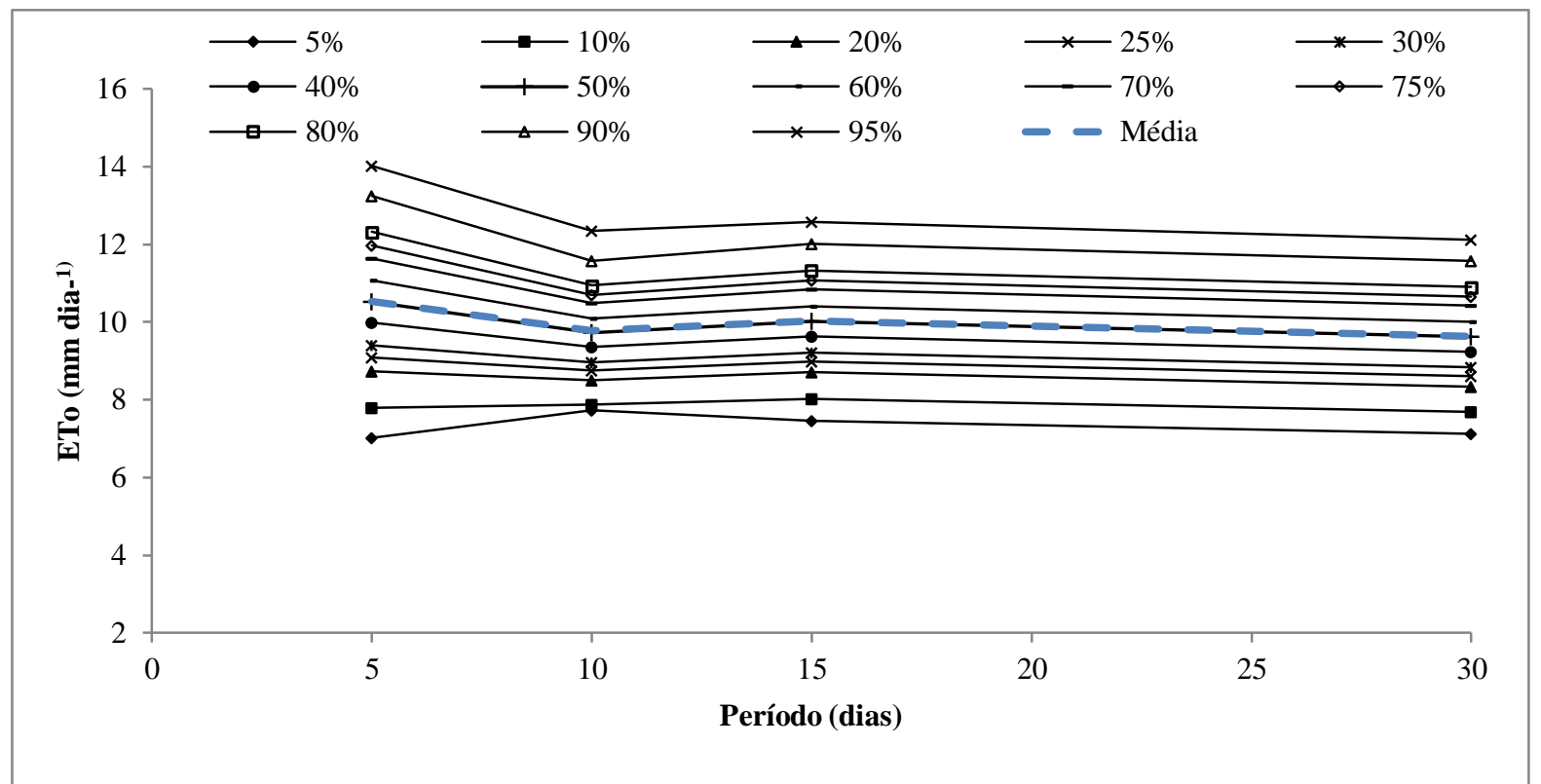

Figura 2. Evapotranspiração de referência $(\mathrm{ETo}, \mathrm{mm})$ para os diferentes níveis de probabilidade de ocorrência para a distribuição normal em função da duração do período para o mês de outubro. 
A. O. da Silva et al.

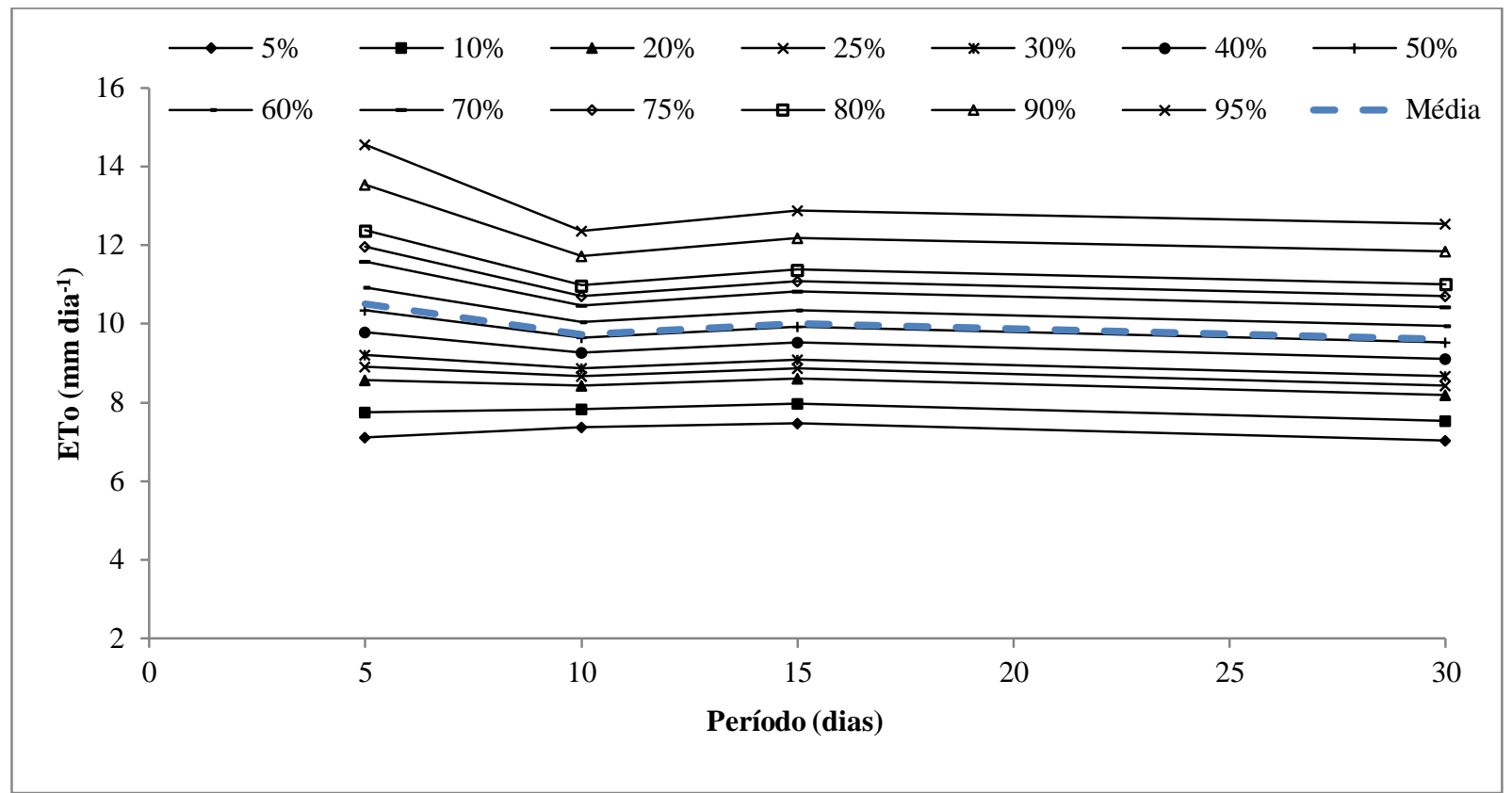

Figura 3. Evapotranspiração de referência $(\mathrm{ETo}, \mathrm{mm})$ para os diferentes níveis de probabilidade de ocorrência para a distribuição gama em função da duração do período para o mês de outubro. 